\title{
Diffuse cutaneous melanosis: rare complication of metastatic melanoma*
}

\author{
Ana Cristina Vervloet do Amaral ${ }^{1}$ \\ Elton Almeida Lucas ${ }^{3}$
}

\author{
Lucia Martins Diniz ${ }^{2}$ \\ Rafaela Lorenzon de Aragão Capeli ${ }^{4}$
}

DOI: http:/ / dx.doi.org/10.1590/abd1806-4841.20176097

\begin{abstract}
Diffuse cutaneous melanosis is a rare complication of metastatic melanoma related to a worse prognosis. There are few cases reported in the literature. Its pathogenesis has not been completely elucidated, although studies have suggested certain mechanisms for its occurrence. It is clinically manifested as a blue-gray discoloration of the skin and mucous membranes in a cephalo caudal progression and usually associated with melanuria. Skin and mucosa histopathology reveals only the presence of melanophages in the dermis, mainly perivascular, and free interstitial melanin. We report the case of a 68-year-old male with metastatic melanoma, diffuse hyperpigmentation of the skin and melanuria.
\end{abstract}

Keywords: Melanosis; Melanoma; Paraneoplastic syndromes

\section{INTRODUCTION}

Diffuse cutaneous melanosis (DCM) is a rare complication of advanced metastatic melanoma, with few reported cases in the medical literature since it was first described by Wagner in $1864 .{ }^{1-6}$

It is characterized by progressive and diffuse blue-gray hyperpigmentation of the skin and mucous membranes, secondary to the abnormal deposit of melanin in the dermis. ${ }^{1-5}$ Histopathology findings consist in the deposition of perivascular histiocytes filled with melanin and free pigment in the dermal connective tissue. ${ }^{1-3,6}$ The gray color of the skin is due to the depth where the melanin is found. Rarely, melanoma cells are seen in the skin and there are few reports of increased melanin and melanocytes in the skin. ${ }^{1-3}$ The dark urine color - melanuria - is a frequently associated finding with this condition..$^{1,2,4}$ Other manifestations besides cutaneous, mucosal and urine pigmentation, such as darkening of hair, of the peritoneal fluid, sputum and internal organs can also be found. ${ }^{1,2}$
We report the case of a patient with diffuse melanoderma and history of an advanced melanoma removed six years prior, with no previous diagnosis.

\section{CASE REPORT}

A 64-year-old man, farmer, Fitzpatrick phototype I, presented with diffuse gray-blue coloration of the skin, with a cephalocaudal progression over the past three months (Figure 1). There was also history of weight loss $(7 \mathrm{Kg})$ in seven months, dyspnea, cough and dark urine (Figure 2). On physical examination, the abdomen was enlarged and there was hepatomegaly. He had no comorbidities. As past history, a pigmented skin lesion had been excised from the left lumbar region six years prior, in another hospital, but he had no knowledge of the histopathology.

\footnotetext{
Study submitted on 31.05.2016

Approved by the Advisory Board and accepted for publication on 16.10.2016

* Study conducted at Universidade Federal do Espírito Santo (UFES) - Vitória (ES), Brazil.

Financial support: None

Conflict of interest: None

Private practice - Vitória (ES), Brazil.

Department of Dermatology of the Universidade Federal do Espírito Santo (UFES) - Vitória (ES), Brazil.

Department of Pathology of the Universidade Federal do Espírito Santo (UFES) - Vitória (ES), Brazil.

Private practice - Brasília (DF), Brazil.
} 
The patient was admitted to the gastroenterology ward at the university hospital for investigations. Chest radiography and chest and abdomen computed tomography were performed, that showed multiple nodular images in the lungs and liver (Figure 3). Laboratory tests detected abnormalities in the liver enzymes (AST $=$ $98 \mathrm{U} / \mathrm{L}, \mathrm{ALT}=196 \mathrm{U} / \mathrm{L}, \mathrm{AF}=1724 \mathrm{U} / \mathrm{L}, \mathrm{GGT}=462 \mathrm{U} / \mathrm{L}$ ) and raised LDH (817 U/L). Colonoscopy demonstrated melanosis colis, with no other abnormalities. A skin biopsy was performed in the area of hyperpigmentation on the face, and histopathology showed perivascular melanocytes in the superficial and deep dermis, and liver biopsy showed melanoma metastases (Figures 4 and 5).

The patient was discharged and referred to an outpatient clinic of Oncology at a tertiary hospital, but died in less than 1 month.

\section{DISCUSSION}

DCM is a rare condition associated to metastatic melanoma. According to a systematic world literature review, 70 cases of this condition were published. ${ }^{1}$ Data from the systematic review show

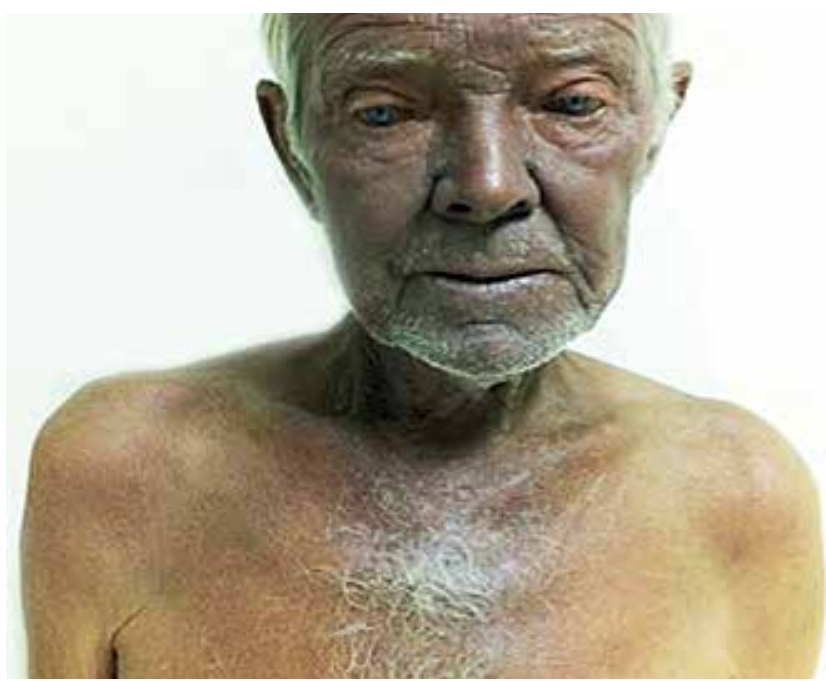

Figure 1: Diffuse gray-blue color of the skin, predominantly on photo-exposed areas

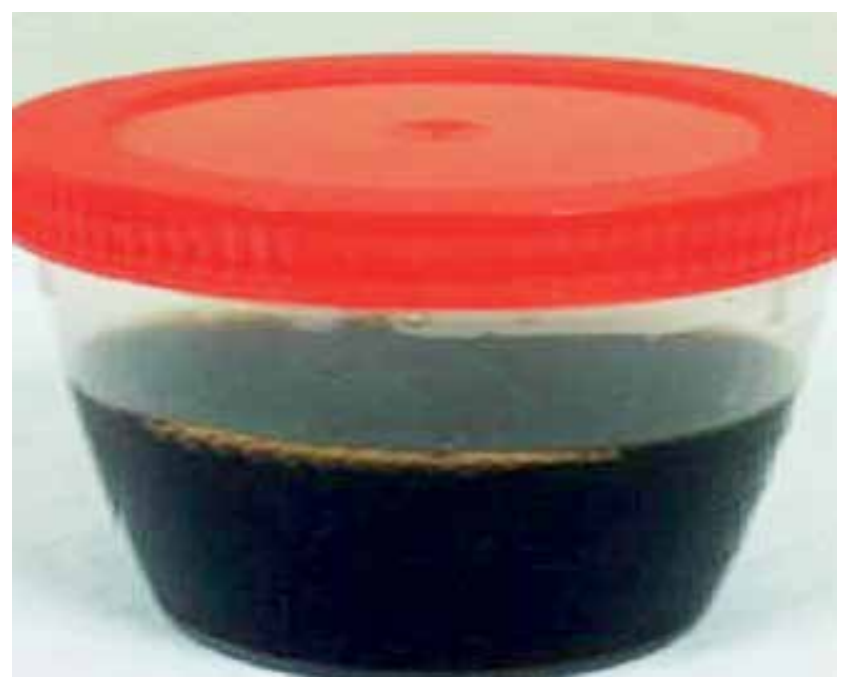

FIGURE 2: Melanuria that men are more commonly affected than women, with $60 \%$ of cases. ${ }^{1}$ Most patients were Caucasian (95\%) and mean age at diagnosis was of 50 years and 4 months. ${ }^{1}$ The back was the most commonly affected site for the primary melanoma (50\%), and the association with melanuria was described in $77 \%$ of cases. ${ }^{1}$ Two reports described melanuria in about $15 \%$ of cases. ${ }^{2,4}$

The case reported has epidemiological (white male) and clinical (site of the primary lesion, predilection of the melanosis for photo-exposed, melanuria and survival) features similar to those found in all publications.

The physiopathogenesis of DCM has not been completely stablished. . $2,4,6,7$ The most accepted theory is that metastatic mela-

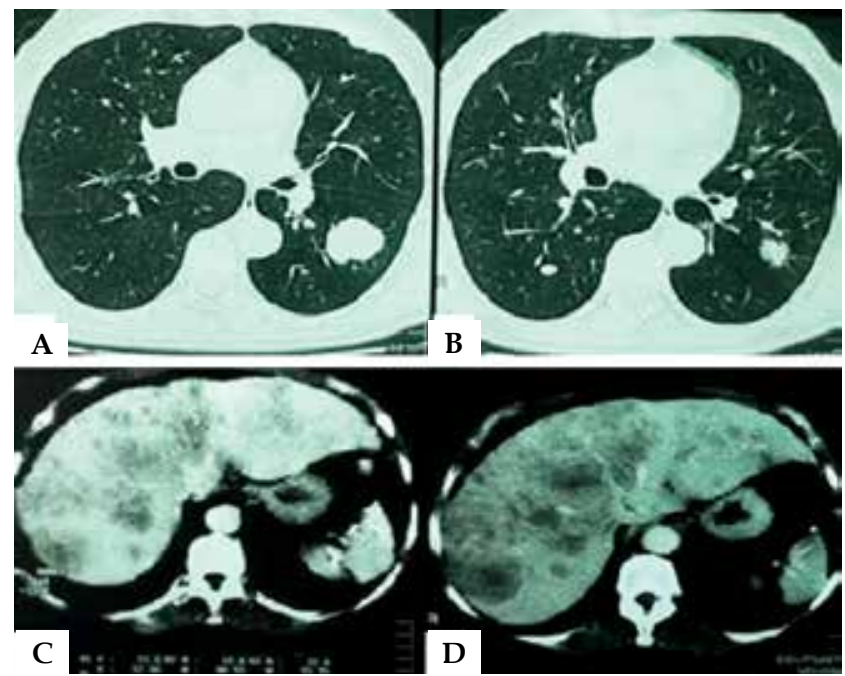

Figure 3: Chest and abdomen computed tomography. A and B: Multiple nodules in the lungs and a mass in the left inferior lobe. C and D: Multiple nodules in the liver
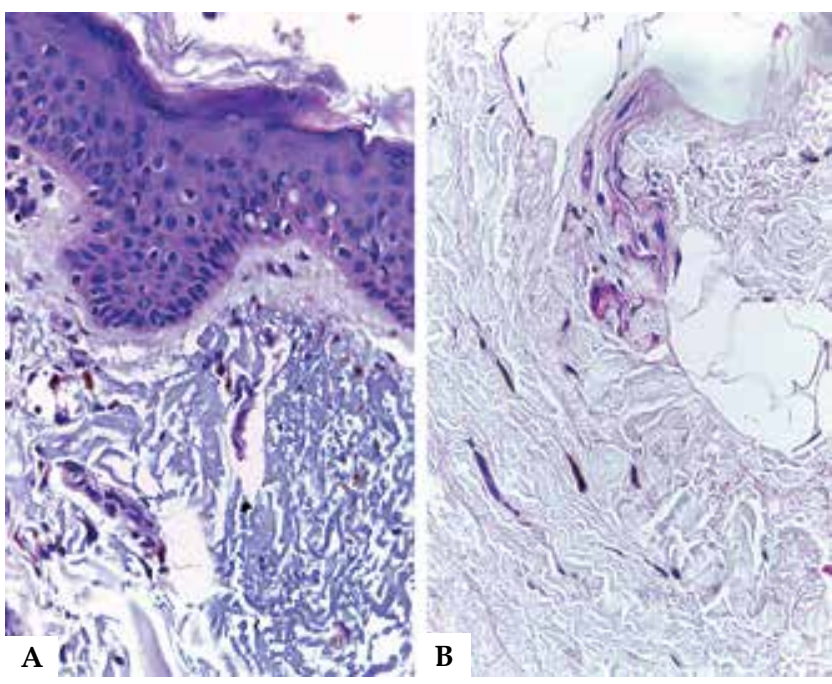

Figure 4: Skin histopathology. A: Perivascular and interstitial melanocytes in the dermis (Hematoxylin \& eosin, X40). B: Interstitial melanophages in the deep dermis, adjacent to subcutaneous tissue (Hematoxylin \& eosin, X40) 


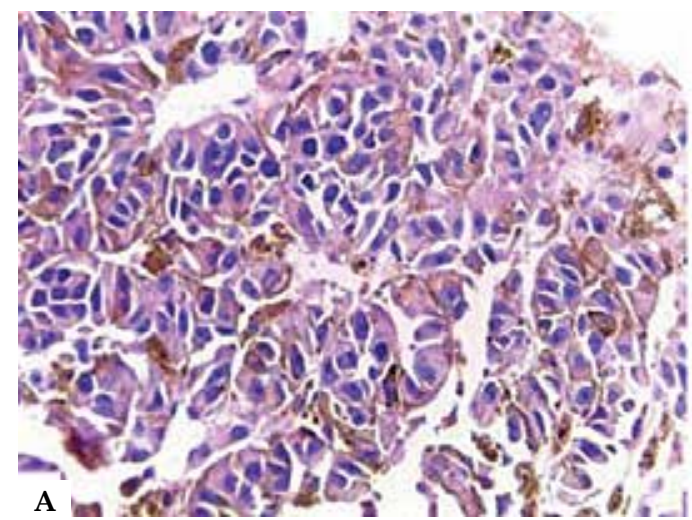

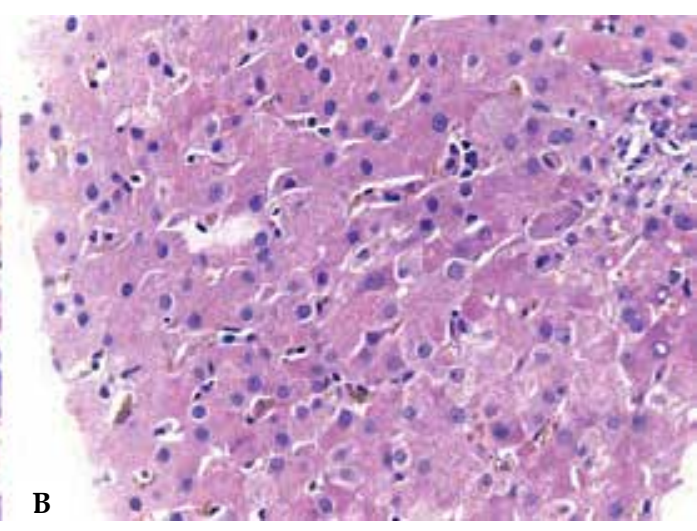

Figure 5:

Liver histopathology. A and B: Cells of metastatic melanoma among hepatocytes and melanin in the liver sinusoids (Hematoxylin \& eosin, X40) noma undergoes cytolysis by central ischemia, immunological responses or oncologic treatments, releasing great amounts of melanin precursors, free melanin and melanosomes. ${ }^{1,3}$ Melanin precursors are released into the blood stream, where they can be converted into melanin by serum oxidants or phagocytosed and processed by macrophages/histiocytes, and converted into melanin by the action of lysosomes. ${ }^{1,3,4}$ When lodged in the dermis, the consequence is melanosis. Melanophages are eventually degraded on the site, forming residues of melanosomes and melanin that also contribute to melanosis. ${ }^{1,3}$ Circulating melanin precursors are capable of invading the glomeruli and be excreted in urine, explaining melanuria, which becomes more marked after exposure to oxygen in the environment, due to the oxidation of melanin. ${ }^{1,2}$ The melanin buildup in the renal tubules and glomeruli endothelial cells can lead to renal dysfunction and increase in the nitrogenous compounds. ${ }^{2}$

The perivascular distribution of histiocytes containing pigment in their interior supports the theory that these melanophages come from the circulation instead of originating locally. ${ }^{1}$ The progressive and diffuse nature of DCM suggests a continuous influx of pigment in the blood stream. Its cephalocaudal progression and predilection for phot-exposed areas are well described in the literature. ${ }^{1}$

Patients with DCM have a poor prognosis, with an average survival of four to six months after the onset of pigmentation. ${ }^{1,2,4}$

This case report is justified by the rarity of this kind of clinical presentation of the melanoma and for the fact that the authors found only one case in the literature reported by Brazilians. $\left.{ }^{8}\right]$

\section{REFERENCES}

1. Sebaratnam DF, Venugopal SS, Frew JW, McMillan JR, Finkelstein ER, Martin LK, et al. Diffuse melanosis cutis: A systematic review of the literature. J Am Acad Dermatol. 2013;68:482-8.

2. Gambichler T, Stücker M, Kerner K, Weiner $\mathrm{S}$, Waldherr R, Altmeyer $\mathrm{P}$, et al. Acute kidney injury in a patient with melanuria, diffuse melanosis, and metastatic melanoma. Am J Clin Dermatol. 2008;9:267-70.

3. Busam KJ, Wolchok J, Jungbluth AA, Chapman P. Diffuse melanosis after chemotherapy-induced tumor lysis syndrome in a patient with metastatic melanoma. J Cutan Pathol. 2004;31:274-80.

4. Perez A, Turajic S, Szyszko T, O'Doherty M, Calonje E, Harries M, et al. Generalized melanosis and melanuria in a patient with metastatic melanoma. Clin Exp Dermatol. 2010;35:e37-9.

5. Sebaratnam DF, Martin LK, Venugopal SS, Mangos G, Freiman J, de Souza P, et al. Diffuse melanosis cutis in the setting of BRAF (V600E) metastatic melanoma. Int J Dermatol. 2014;53:1409-11.

6. Tsukamoto K, Furue M, Sato Y, Takayama 0, Akasu R, Ohtake N, et al. Generalized melanosis in metastatic malignant melanoma: the possible role of DOPAquinone metabolites. Dermatology. 1998;197:338-42.

7. Sendagorta E, Pizarro A, Feito M, Mayor M, Ramírez P, Floristan U, et al. Diffuse melanosis and ascites due to metastatic malignant melanoma. ScientificWorldJournal. 2008;8:556-7.

8. Paulo Filho Tde A, da Trindade Neto PB, Reis JC, Bartelt L, da Costa SA.. Diffuse cutaneous melanosis in malignant melanoma. Dermatol Online J. 2007;13:9.

\author{
MAILING ADDRESS: \\ Ana Cristina Vervloet do Amaral \\ Avenida Marechal Campos, 1355 \\ Santos Dumont \\ 29041-295 Vitória, ES \\ Brazil \\ E-mail: anavervloet@hotmail.com
}

How to cite this article: Amaral ACV, Diniz LM, Lucas EA, Capeli RLA. Diffuse cutaneous melanosis: rare complication of metastatic melanoma. An Bras Dermatol. 2017;92(5 Suppl 1): 62-4. 